[Frontiers in Bioscience S3, 226-235, January 1, 2011]

\title{
Periacinar retraction artifact of the prostate
}

Bozo Kruslin ${ }^{1,2}$, Davor Tomas ${ }^{1,2}$, Gregor Mikuz ${ }^{3}$

${ }^{1}$ Ljudevit Jurak Department of Pathology, Sestre milosrdnice University Hospital, Vinogradska 28, 10 000 Zagreb, Croatia, ${ }^{2}$ University of Zagreb, School of Medicine, Salata 3, 10000 Zagreb, Croatia, ${ }^{3}$ Department of Pathology, Medical University Innsbruck, Mullerstrase 44, A-6020 Innsbruck, Austria

\section{TABLE OF CONTENTS}

\author{
1. Abstract \\ 2. Introduction \\ 3. Retraction artifact in diagnosis of prostate carcinoma \\ 4. Prognostic significance of retraction artifact in prostate carcinoma \\ 5. Possible origin of retraction artifact in prostate carcinoma \\ 6. Conclusions and perspectives \\ 7. References
}

\section{ABSTRACT}

Retraction artifacts are well known phenomenon in diagnostic surgical pathology for a long time but they were usually considered as artificially produced tissue alteration. Some recent studies of retraction artifact in different tumors have focused on its diagnostic and/or prognostic role. Their presence in prostatic carcinoma may be used in diagnostic and prognostic purposes. Peritumoral retraction artefacts in prostatic carcinoma are more pronounced and more common around neoplastic glands compared to benign glands, gland with prostatic intraepithelial neoplasia and postatrophic hyperplasia that is the one of the most common mimickers of prostatic carcinoma. In addition, the presence of extensive retraction artifact in prostatic carcinomas is clearly associated with tumor features that indicate a more aggressive tumor phenotype and with shorter biochemical recurrence-free survival. Studies at the molecular level demonstrate that retraction artifact may be attributed to molecules produced by reactive cancer stroma. The origin of retraction artifact in tumor specimens is unknown but they are probably the consequence of lack of basal cells and /or stromal changes but not simply artifacts due to laboratory procedures.

\section{INTRODUCTION}

Retraction artifacts are well known phenomenon in diagnostic surgical pathology for a long time but have received very little attention in routine daily practice. They were usually considered as artificially produced tissue alteration that interferes with the ability to make an appropriate diagnosis. Peritumoral retraction artifact appears in tissue sections as an empty space partially or completely encircling a nest of tumor cells, usually in conformity with the rounded or angular outline of that particular nest. Different terms were proposed for this phenomenon including peritumoral clefting, periacinar halos, retraction clefting or cleft-like spaces $(1,2)$.

Recent investigations have stressed their diagnostic and prognostic significance in different tumors (1-13). Periacinar retraction clefting was proposed as an additional and helpful diagnostic criterion in prostate cancer (1-5), breast carcinoma (6), and urothelial carcinoma (especially in cases of urothelial carcinoma with microinvasion) (7-9). Furthermore, retraction artifact could serve as diagnostic criterion for distinguishing noninvasive and invasive extraovarian implants in borderline serous 


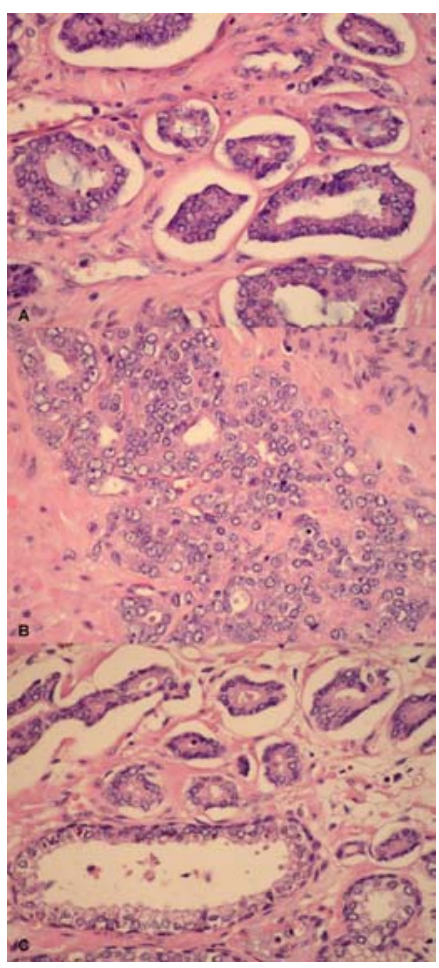

Figure 1. A) Periacinar retraction clefting affecting more than $50 \%$ of circumference of neoplastic acini in prostatic adenocarcinoma (HE, x400). B) Neoplastic acini in prostatic adenocarcinoma without retraction clefting (HE, $\mathrm{x} 400$ ). C) Periacinar retraction clefting is visible around neoplastic glands but not around benign glands that are present in the same area (HE, $\mathrm{x} 400)$.

ovarian tumors. The presence of retraction artifact around solid epithelial nests in extraovarian implants was a sign of invasiveness (10). Retraction clefting may also be observed in other tumor types and can be useful in the differential diagnosis between basal cell carcinoma and adnexal skin tumors (11).

Beside their diagnostic significance, some studies of retraction artifact in tumors have also showed its prognostic role. The presence and/or extent of peritumoral clefting was significantly associated with adverse clinicopathologic tumor features and poor prognosis in borderline serous ovarian tumors with extraovarian implants, breast carcinoma, squamous cell carcinoma of the esophagus and prostate carcinoma (10, 12-15).

Although frequently present in sections of formalin-fixed, paraffin-embedded tissue samples, the origin of and the biologic mechanisms responsible for the retraction artifact or their biologic or clinical significance are still largely unresolved.

\section{RETRACTION ARTIFACT IN DIAGNOSIS OF PROSTATE CARCINOMA}

Prostatic adenocarcinoma is the most common noncutaneous malignant neoplasm in humans with an estimated prevalence of over 30 percent in males older than 50 years based on histological autopsy studies (16). The diagnosis of prostatic carcinoma is complex, especially in needle core biopsies and is based on a constellation of three major histological criteria: the infiltrative growth pattern, the absence of a basal cell layer and the presence of macronucleoli (nucleoli larger than 1 micrometre in diameter) $(1,16)$. There are also three strong additional histologic criteria that are considered as diagnostic for prostatic adenocarcinoma including perineural invasion, mucinous fibroplasias (collagenous micronodules) and glomerulations (17). Several supportive diagnostic criteria have been proposed, but only a minority of these supportive features is specific for tumor. Proposed suportive criteria used in routine diagnostic procedure are: marginated nucleoli, multiple nucleoli, wispy, bluish mucinous secretions, intraluminal crystalloids, intraluminal amorphous eosinophilic material, collagenous micronodules (mucinous fibroplasias), glomerulations, peritumoral clefting and others, but many of these supportive criteria may also be present in benign glands or in some nonneoplastic proliferative glandular conditions $(1$, $16,18,19)$.

One of the proposed additional criteria is peritumoral retraction artifact appearing more frequently around neoplastic glands in comparison with benign glands $(1,20-23)$. Halpert et co-workers $(21,22)$ were the first to shortly describe the retraction artifacts in prostatic adenocarcinoma. In their autopsy studies of prostatic adenocarcinoma, halos around tumorous acini were observed. In a study by Varma et al (1) retraction artifacts were found in approximately $40 \%$ of cases of prostatic cancer diagnosed by needle core biopsy and in $7 \%$ of nonneoplastic glands. They graded retraction artifact as a percentage of gland circumference separated from the stroma using a scale from $0-4$. The same authors considered grade $3(50-75 \%)$ and 4 (more than $75 \%$ ) as positive. We analyzed retraction artifact under high magnification $(\mathrm{x} 40$ objective) counting 10 glands in three different fields (2). Retraction artifact affecting more than $50 \%$ of circumference in more than $50 \%$ of analyzed glands was found in neoplastic glands only. Such strict criteria were fulfilled in about $16 \%$ of all cancer cases and were not observed in normal glands. The application of less stringent criteria (one third of glands with more than $50 \%$ of circumference) revealed positive retraction artifact in $51.8 \%$ of carcinoma cases and $8 \%$ of nonneoplastic glands that was also statistically significant. Similar retraction artifact changes should not be present in adjacent normal glands within the same specimens (Figure $1 \mathrm{~A}, \mathrm{~B}$ and $\mathrm{C}$ ). Retraction artifact could be observed also under lower magnification. Our criteria may be applied in a similar manner to carcinoma cases composed of few neoplastic glands (2).

The presence of retraction artifact in prostatic needle core biopsy was usually attributed to the inadequate laboratory procedure. Therefore, the quantity of retraction artifact was considered as an indicator of quality of laboratory staff. To minimize the influence of laboratory procedure and different technicians an improved 


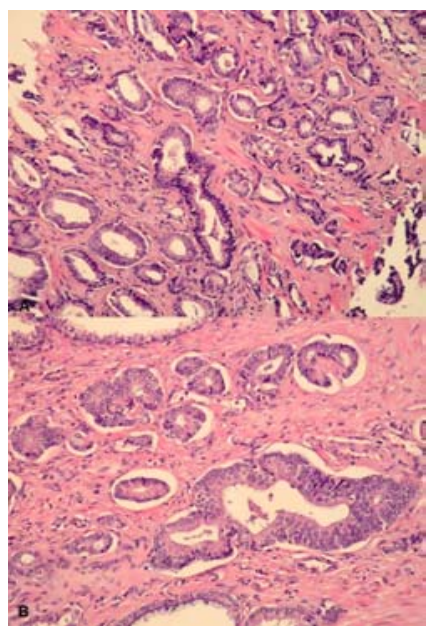

Figure 2. Periacinar retraction clefting affecting more than $50 \%$ of circumference of neoplastic acini in prostatic adenocarcinoma in A) needle core biopsy and B) matched radical prostatectomy specimen (HE x 100)

preembedding method of prostatic needle core biopsy specimens was employed (24-26).

Irie et al (6) showed that peritumoral retraction artifact seen during the evaluation of hematoxylin and eosin specimens was a significant finding for the diagnosis of invasive breast carcinoma. They found peritumoral retraction artifact in 168 of 199 cases $(84.4 \%)$ of invasive ductal carcinoma, versus 30 of 188 cases $(16 \%)$ of ductal carcinoma in situ. Peritumoral retraction artifact that affected more of $26 \%$ tumor nests was seen in only 1 of 188 ductal in situ carcinoma specimens, compared with 77 of 199 invasive ductal carcinoma (6).

McKenney et al (7) assessed the morphological features of early and focal invasion associated with urothelial carcinoma in situ in a series of 77 biopsy and cystectomy bladder specimens. The common patterns found were irregular nests, cords extending from the overlying urothelium, single cells invading the lamina propria and retraction artifact around clusters or single invasive cells, which mimics vascular invasion. This characteristic stromal response to microinvasion was fairly common and was noted in almost $80 \%$ of cases with microinvasion. Similar retraction artifacts were also previously seen in early invasion associated with papillary neoplasia and in the micropapillary variant of urothelial carcinoma $(8,9)$.

Bell et al (10) proposed retraction artifact as a diagnostic criterion for distinguishing noninvasive and invasive extraovarian implants in borderline serous ovarian tumors. They considered the presence of retraction artifact around solid epithelial nests in extraovarian implants to be a sign of invasiveness. In their study, $83 \%$ of invasive implants had solid epithelial nests surrounded by retraction artifact and the presence of retraction artifacts strongly correlated with adverse outcome (10).
We have further analyzed retraction artifacts in prostatic adenocarcinoma in needle core biopsies and corresponding tumor after prostatectomy to contribute to refinement of the criteria for the determination of retraction artifact and to confirm its diagnostic value (3). There was a significant correlation if the criterion was one third or more glands with retraction artifact affecting more than $50 \%$ of circumference. A stricter criterion that regarded as positive the cases with at least $50 \%$ of neoplastic glands (15 or more of 30) with retraction artifact that affected more than $50 \%$ of circumference showed retraction artifact in $24(15.8 \%)$ of needle biopsies and $37(24.3 \%)$ of prostatectomy material. (3). There was a correlation of periacinar retraction artifact between needle core and corresponding prostatectomy specimens suggesting that retraction artifacts are not simple artifacts caused by laboratory procedure but in fact may represent a reliable criterion for diagnosis of the prostatic adenocarcinoma (Figure 2A and B). Similar results regarding correspondence of periacinar retraction artifact in needle core biopsies and larger surgical specimens were also reported by Acs et al (12) for breast carcinoma.

There are many different benign conditions that may mimic prostatic carcinoma, like partial and/or complete atrophy, adenosis and many others (16, 27-30). According to recent studies, one of the most common mimickers is postatrophic hyperplasia (PAH) $(27,28)$. $\mathrm{PAH}$ is characterized by hyperplastic glands intermingled with atrophic ones lined with cells with scanty cytoplasm. The degree of nuclear atypia is less than in most carcinomas. The slit-like acini and apical blebs of some cases of PAH are very rare in most carcinomas $(16,27,28)$. The dense fibrotic stroma and shrunken muscle cells that may be seen in PAH are quite uncommon in carcinoma. An obvious infiltrative growth pattern is not seen in PAH and lobulation, which is usually present, is helpful architectural feature $(27,28,31)$. Adjacent typical atrophy may be a clue to the diagnosis. The basal cell layer is typically present, although it may be difficult to demonstrate it, in some cases even by immunostaining using HMW- cytokeratin or p63 $(16,32)$.

Numerous features that might help in the differential diagnosis between atrophy and carcinoma are described $(16,27,28)$. The presence of retraction clefting around neoplastic glands is an additional criterion favoring prostatic adenocarcinoma (5). Foci of atrophic glands may be classified in two main groups; proliferative atrophy (PA) and proliferative inflammatory atrophy (PIA) and each group may be subclassified into simple atrophy (SA) and postatrophic hyperplasia (PAH), as proposed by the working group for histologic classification of focal prostate atrophy lesions (31). Our study showed no significant difference in the presence and extent of retraction clefts between PA and PIA (5). Prostatic carcinoma showed statistically significant difference in the frequency and extent of retraction clefts, as compared to PA and PIA (Figure $3 \mathrm{~A}$ and $\mathrm{B}$ ). According to our knowledge and data from the literature it was not routinely applied to distinguish atrophy from carcinoma (1-3). 


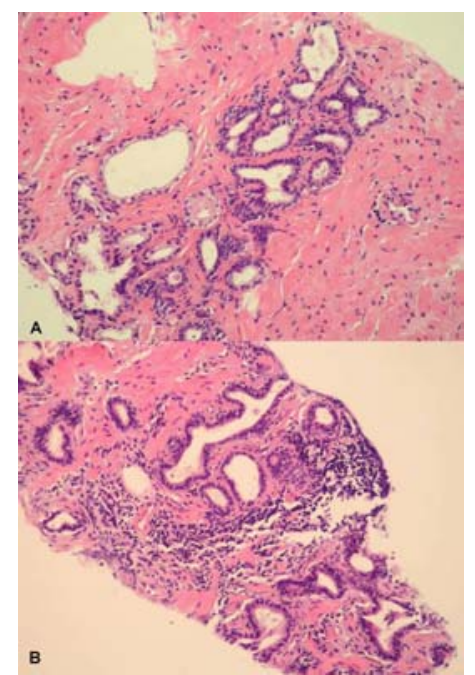

Figure 3. Atrophic glands in focuses of proliferative atrophy (A) and proliferative inflammatory atrophy (B) were mostly without periacinar clefting or clefting was present in less than half of the gland circumference (HE, $\mathrm{x} 200)$.

\section{PROGNOSTIC SIGNIFICANCE OF RETRACTION ARTIFACT IN PROSTATE CARCINOMA}

Some recent studies of retraction artifact in tumors have focused on its prognostic role $(10,12-14)$. The presence and/or extent of peritumoral clefting was significantly associated with adverse clinicopathologic tumor features and poor prognosis in borderline serous ovarian tumors with extraovarian implants, breast carcinoma and squamous cell carcinoma of the esophagus $(10,12-14)$.

We examined whether the presence and extent of peritumoral retraction artifact could be used to predict biochemical recurrence-free survival in prostatic carcinoma. We also analyzed the correlation between the extent of retraction artifact in the tumors and various clinicopathologic tumor features (15).

Our results revealed that the retraction artifact in prostatic carcinoma correlated with different clinicopathologic tumor features and biochemical recurrence-free survival. Patients with high preoperative PSA and short follow-up time had tumors with a significantly higher percentage of retraction artifacts. While a minimal inclusion criterion for patients without relapse was a follow-up of 60 months, many of the patients with biochemical relapse were included in the study despite a shorter follow-up time. Thus, negative correlation of follow-up time and extent of the retraction artifact indicated that tumors in patients with biochemical recurrence harbored more extensive retraction artifacts. Seminal vesicle invasion and/or extracapsular extension of the tumor (T3 stage tumors) and positive surgical margins were also significantly more common in tumors with extensive retraction artifacts. The extent of retraction artifact showed no significant correlation with lymph node metastasis, but any possible conclusions in this regard are hampered by the fact that only six patients in our study group had nodal metastasis, which is an insufficient number for a reliable statistical analysis. In the group of 46 patients with biochemical recurrence extent of retraction artifact was not significantly different and was not significantly associated with disease free survival time. This fact could indicate that the extent of retraction artifact over some cutoff value had no further implications on biochemical recurrence and disease free survival time. In our investigation, cutoff value was set on retraction artifact involving at least $20 \%$ of the tumor glands. The presence of extensive retraction artifact in prostatic carcinomas was clearly associated with tumor features that indicated a more aggressive tumor phenotype. In addition, extensive retraction artifact was associated with poor biochemical recurrence-free survival in both univariate and multivariate analyses (15).

Similar prognostic significance of retraction artifact was reported for breast carcinoma $(12,13)$. In the study of Acs et al (12) the extent of retraction artifacts showed a significant correlation with increasing tumor size, histologic type, combined histologic grade, and all three components of the histologic grading system. Tumors associated with lymphatic vessel invasion and axillary lymph node metastases showed significantly higher amounts of retraction artifacts compared with tumors without these features. Further, extensive retraction artifact in breast carcinoma was significantly associated with poor overall and disease-free survival (12).

In a more recent study, Acs et al (13) also showed that the presence of extensive retraction artifacts in core needle biopsy samples of invasive ductal carcinoma was significantly associated with the presence of lymph vessel invasion and lymph node metastasis. These findings indicated that the presence of extensive retraction artifacts on core needle biopsy can predict nodal metastasis in the breast carcinoma (13).

In esophageal squamous cell carcinoma, the presence and the extent of retraction artifacts was associated with local tumor penetration and lymph node metastasis. Tumors with extensive retraction artifact mostly presented at a higher stage and with lymph node metastasis. Thus, extensive retraction artifact pertained to a more aggressive tumor behavior and could be viewed as a simple and useful morphological feature of tumor aggressiveness (14).

In addition, as we previously mentioned, Bell et al (10) showed that presence of retraction artifact around solid epithelial nests in extraovarian implants of borderline serous ovarian tumors strongly correlated with adverse outcome (10).

\section{POSSIBLE ORIGIN OF RETRACTION ARTIFACT IN PROSTATE CARCINOMA}

The origin of retraction artifact in tumor specimens is unknown. Few hypotheses should be taken in 


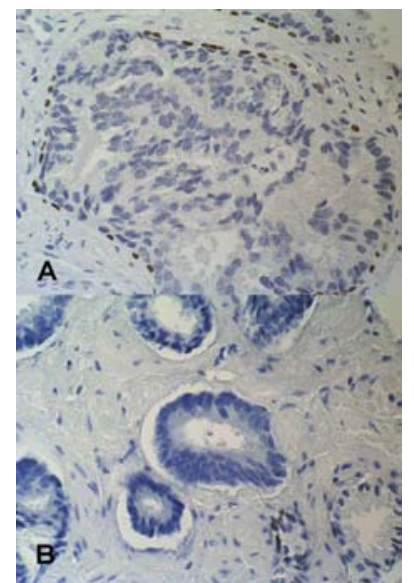

Figure 4. P63 immunostaining in prostatic intraepithelial neoplasia (A) and prostatic adenocarcinoma (B). Nonneoplastic glands served as a positive control (p63, $\mathrm{x} 400)$.

consideration. First, malignant glands in some carcinoma such as prostatic and breast carcinoma lack basal cells and this could be one of the causes for appearance of retraction artifact. Second, appearance of specific reactive cancer stroma may disorder maintenance of normal prostatic stromal-epithelial homeostasis, and could play a role in the retraction artifact formation (33). Third, different molecules produced by reactive cancer stroma or molecules involved in cell adhesion secreted by epithelial cancer cells could also be implicated. Fourth, some authors proposed retraction artifact to be prelymphatic spaces and their appearance connected with lymph vessels invasion (6).

To analyze hypothesis that retraction artifact is probably connected to the lack of basal cells we correlated the presence and extent of retraction artifact and the expression of p63 in neoplastic glands and glands with prostatic intraepithelial neoplasia (PIN) in needle core biopsies (4). PIN is the most likely precursor of prostatic adenocarcinoma according to available data from the literature $(18,34,35)$. PIN is the abnormal proliferation within the prostate ducts, ductules and large acini of premalignant foci of cellular dysplasia and carcinoma in situ without stromal invasion $(18,34,35)$. There is preservation of the basal cell layer and basement membrane, however focal disruption of basal cell layer may be observed (35). The incidence of PIN varies according to type of bioptic material. PIN is associated with progressive abnormalities of phenotype and genotype (35, 36). The ability of immunohistochemical staining to detect basal cells has proven to be diagnostically extremely valuable, especially in needle biopsy specimens. Antibodies against high molecular weight cytokeratin (34betaE12) and p63 are frequently used basal cell markers to aid in the diagnosis of prostatic carcinoma (37). Zhou et al (38) recommended this basal cell cocktail for routine prostatic carcinoma diagnostic work-up. Technical factors such as tissue fixation and antigen retrieval techniques may also make the detection of basal cells difficult $(37,38)$.
P63, a homologue of the tumor suppressor gene $\mathrm{p} 53$, has been shown in the basal cell component of epithelium from a variety of tissues, including prostatic epithelium (39-41). Value of p63 immunostaining in the diagnosis of prostatic adenocarcinoma has been clearly shown $(40,41)$. However, the presence of clearly identifiable basal cells in a gland or duct does preclude the diagnosis of carcinoma for that structure (39). At least focal high molecular weight cytokeratin (clone34betaE12 antibody) positivity was observed in $1.1 \%$ of 3198 prostatic carcinoma cases diagnosed on needle core biopsies (39).

Our investigation performed on 28 core biopsy with PIN and 41 core biopsy with prostatic carcinoma showed that periacinar retraction clefting was significantly more prominent in prostatic carcinoma compared to PIN and nonneoplastic glands. There was no difference between normal glands and PIN regarding clefting. P63 was positive around whole circumference in 12 out of 28 cases with $\mathrm{PIN}$, and discontinuously positive in remaining 16 PIN cases suggesting initial disruption of basal cell layer (Figure 4A and B). P63 immunostaining was also positive in all nonneoplastic glands, and negative in all carcinoma cases. These data suggest that retraction clefting was associated with cancer and lack of basal cells, but not with PIN (4).

Reactive stromal changes that occur in different human cancers are probably involved in local tumor spreading. It seems that stromal components play very important role in enhancement of tumor progression by stimulating angiogenesis and by promoting cancer cell survival, proliferation, and invasion. Studies of human breast, colon and prostatic cancer specimens have identified activated stromal cell phenotypes, modified extracellular matrix (ECM) composition, and increased microvessel density, exhibiting biological markers consistent with stroma at site of wound repair (42-45).

It is well known that stromal cells modulate normal prostatic development, growth, and differentiation through stromal-epithelial interactions $(46,47)$. In the normal prostate, prostatic smooth muscle cells under the influence of androgen signal to prostatic epithelium control epithelial differentiation and suppress epithelial proliferation. On the other hand, prostatic epithelium sends signals to prostatic smooth muscle cells to maintain smooth muscle differentiation (48-50). In experimental models, prostate stromal cells promote angiogenesis and stimulate the development and rate of human prostate tumorigenesis $(51,52)$. Furthermore, it is well known that stromal changes in prostatic carcinoma could have implication on prognosis of the disease $(53,54)$. Ayala et al (53) and Tomas et al (54) showed that intensity of stromal changes could serve as an independent prognostic factor in the assessment of biochemical recurrence-free survival in patients with prostatic carcinoma that underwent radical prostatectomy. In addition, intensity of stromal changes could identify patients with higher risk of disease recurrence in cases of identical Gleason score (54). 
Ayala et al (53) and Yanagisawa et al (55) also showed that volumes of reactive stroma could predict biochemical recurrence-free survival in prostatectomy specimens and needle core biopsies. Tumors with no reactive stroma and tumors with abundant reactive stroma had a worse prognosis compared to tumors with a moderate quantity of stroma. They explained these findings by two theories. Firstly, the tumors with no stroma had a worse prognosis because in these tumors epithelial cells began to express genes that are normally restricted to stromal cells and became stroma-independent, with a consequent decrease in stroma quantity. Secondly, the tumors with a large amount of reactive stroma had a worse prognosis because abundant stroma could produce larger quantities of growth factors and also could serve as a shield from host immune and inflammatory response $(53,55)$.

In normal prostate, stroma is predominantly composed of smooth muscle cells with very few fibroblasts, myofibroblasts and collagen fibers. Reactive stroma in cancer is composed of fibroblasts, myofibroblasts, endothelial cells, and immune cells and shows decrease in number of smooth muscle cells (45).

Tuxhorn et al (46) histochemically and immunohistochemically showed increased number of stromal myofibroblasts in prostatic cancer by double-label flourescent immunohistochemistry. Myofibroblasts as well as peritumoral clefting were most prominent in Gleason grade 3 prostatic carcinoma $(46,47)$. These findings confirmed that (myo)fibroblastic stromal changes characterized cancer reactive stroma. Cancer growth subsequently leads to increased amount of (myo)fibroblasts and decreased amount of smooth muscle cells as cancer growth progresses. No similar changes were observed in adjacent peritumoral stroma, what was confirmed by similar staining pattern on Mallory method, and similar vimentin, alpha-SMA and desmin expression compared to prostatic hyperplasia (47).

Myofibroblasts in reactive stroma synthesize ECM components such as collagen I, collagen III, fibronectin, tenascin, and versican. In addition, myofibroblasts express proteases, FAP (fibroblast activation protein), and MMPs (matrix metalloproteinases). These products are remodeling ECM and basement membrane and could stimulate cancer cell growth and migration (56). They also stimulate angiogenesis secreting VEGF (vascular endothelial growth factor), especially VEGF-D. VEGFs are expressed in myofibroblasts under hypoxia or in response to growth factors (57). Therefore it seems that retraction artifact may be attributed to some molecules produced by reactive cancer stroma.

Tenascin-C (TN-C) is a large $(180-300 \mathrm{kDa})$, hexameric multidomain glycoprotein located mainly in the ECM that is involved in tissue interactions during embryogenesis, wound heeling, inflammation and oncogenesis (58). The most prominent function of TN-C includes anti-adhesion effects, favouring cell motility and growth promotion. These proposed activities suggest a potential role for $\mathrm{TN}-\mathrm{C}$ in regulation of tumor cells proliferation, invasion and metastasis $(59,60)$.

To become invasive, prostate cancer cell must first penetrate acinar basement membrane (BM) proteins and extracellular matrix adhesive glycoproteins (61). Structural components of the BM are laminins, type IV and type VII collagens, nidogens, and proteoglycans (61).

Laminins are heterotrimeric molecules made up by one alpha, one beta and one gamma chain. Until today we know of five alpha-chains, three beta-chains and three gamma-chains. These chains combine into at least 14 different laminins. The distribution of these laminin isoforms varies between tissues, but in most BMs more than one laminin is present (62).

Laminins are associated with a variety of biological activities such as cell differentiation, cell shape and movement, maintenance of tissue phenotypes, and promotion of tissue survival and their functions in tumor invasion is under extensive research today (63).

We have shown increased immunostaining of tenascin-C, and decreased staining for laminin in carcinomas compared with peritumoral tissue and benign prostate hyperplasia. Gleason pattern 3 carcinomas showed more pronounced stromal reaction and tenascin- $\mathrm{C}$ expression compared with Gleason pattern 4 carcinomas. The main cells in prostate cancer stroma that are responsible for tenascin production are myofibroblasts. Degradation of laminin was not connected with myofibroblastic stromal changes (48). Our study confirmed appearance of myofibroblasts in prostate cancer reactive stroma, especially in Gleason pattern 3 tumors, but not in adjacent peritumourous tissue and stroma in benign prostate hyperplasia. These findings could suggest possible role of tenascin- $\mathrm{C}$ in retraction artifact formation (48).

Our results about tenascin-C expression were consistent with results reported by Tuxhorn and co-workers (46). In their study strong periglandular tenascin expression was also shown in cancer cases. However, they studied tenascin expression on 7 cases of Gleason pattern 3 prostate carcinomas only (46).

Furthermore, molecules secreted by epithelial cells, especially molecules involved in cell adhesion such as Ig superfamily of cell adhesion molecules, integrins, cadherins, selectins, syndecans, epithelial cell adhesion molecule (EpCam) and many others could also be implicated in retraction artifact formation (64-70). Over expression or loss of some of these molecules in cancer epithelial cells could aid in artifact formation.

Irie et al (6) proposed that retraction artifact around tumorous tissue actually represent lymph vessel compartments and their appearance connected with lymph vessels invasion in breast carcinoma. In order to investigate this hypothesis we analyzed relationship between retraction artifact and expression of immunohistochemical marker of lymph vessels D2-40 in prostatic carcinoma (71). Neoplastic glands were analysed in 25 paraffin sections 
from radical prostatectomy specimens with prostatic adenocarcinoma diagnosis. Number of lymph vessels inside tumours tissue ranged between 2 and 75 on the one cross section (average number was 17.4). Lymph vessels that mimicked clefts inside tumours tissue ranged between 0 and 10 on the one cross section (average number was 1.33). In 30 tumorous glands with most extended clefts on each cross section number of lymph vessels ranged between 0 and 3 (average number was 0.6). These results suggest that lymph vessels can mimic retraction artifact inside tumor, but retraction artifacts do not represent lymphatic spaces and some other mechanism is responsible for their formation (71).

\section{CONCLUSIONS AND PERSPECTIVES}

Peritumoral retraction artifacts may be seen in many different types of carcinoma including prostate, breast, skin, ovary, urinary bladder and others. Their presence may be used in diagnostic and prognostic purposes. Peritumoral retraction artifacts are probably the consequence of lack of basal cells and /or stromal changes but not simply artifacts due to laboratory procedure.

The origin of these changes is still unresolved and future studies, particularly at the molecular level, should be performed to better understand this phenomenon.

\section{REFERENCES}

1. M. Varma, M. W. Lee, P. Tamboli, R. J. Zarbo, R. E. Jimenez, P. G. O. Salles and M. B. Amin: Morphologic criteria for the diagnosis of prostatic adenocarcinoma in needle biopsy specimens. Arch Pathol Lab Med 126, 554-561 (2002)

2. B. Kruslin, D. Tomas, H. Rogatsch, I. Novosel, H. Cupic, M. Belicza, O. Kraus and G. Mikuz: Periacinar retraction clefting in the needle core biopsies. An important diagnostic criterion or a simple artifact? Virchows Arch 443, 524-527 (2003)

3. B. Kruslin, D. Tomas, H. Rogatsch, A. Reljic, M. Vucic, D. Balicevic, M. Belicza and G. Mikuz: Correlation of periacinar retraction clefting in needle core biopsies and corresponding prostatectomy specimens of patients with prostatic adenocarcinoma. Int J Surg Pathol 13, 67-72 (2005)

4. B. Kruslin, D. Tomas, A. Cviko, H. Cupic, L. Odak and M. Belicza. Periacinar clefting and p63 immunostaining in prostatic intraepithelial neoplasia and prostatic carcinoma: Pathol Oncol Res 12, 205-209 (2006)

5. M. Ulamec, D. Tomas, C. Ensinger, H. Cupic, M. Belicza, G. Mikuz and B. Kruslin: Periacinar retraction clefting in proliferative prostatic atrophy and prostatic carcinoma. J Clin Pathol 60, 1098-1101 (2007)

6. J. Irie, V. Manucha, O. B. Ioffe and S. G. Silverberg: Artefact as the pathologist's friend: peritumoral retraction in in situ and infiltrating duct carcinoma of the breast. Int J Surg Pathol 15, 53-59 (2007)

7. J. K. McKenney, J. A. Gomez, S. Desai, M. W. Lee and M. B. Amin: Morphologic expressions of urothelial carcinoma in situ: a detailed evaluation of its histologic patterns with emphasis on carcinoma in situ with microinvasion. Am J Surg Pathol 25, 356-362 (2001)

8. M. B. Amin, J. A. Gomez and R. H. Young: Urothelial transitional cell carcinoma with endophytic growth pattern: a discussion of patterns of invasion and problems associated with assessment of invasion in 18 cases. $\mathrm{Am} \mathrm{J}$ Surg Pathol 21, 1057-1068 (1997)

9. M. B. Amin, J. Y. Ro, T. el-Sharkawy, K. M. Lee, P. Troncoso, E. G. Silva, N. G. Ordonez and A. G. Ayala: Micropapillary variant of transitional cell carcinoma of the urinary bladder. Histologic pattern resembling ovarian papillary serous carcinoma. Am J Surg Pathol $18,1224-1232$ (1994)

10. K. A. Bell, A. E. Smith Sehdev and R. J. Kurman: Refined diagnostic criteria for implants associated with ovarian atypical proliferative serous tumors (borderline) and micropapillary serous carcinomas. Am J Surg Pathol 25, 419-432 (2001)

11. G. F. Murphy and D. E. Elder, Non-Melanocytic Tumors of the Skin. Washington, DC: Armed Forces Institute of Pathology, Washington, DC (1991)

12. G. Acs, K. L. Dumoff, L. J. Solin, T. Pasha, X. Xu and P. J. Zhang: Extensive retraction artifact correlates with lymphatic invasion and nodal metastasis and predicts poor outcome in early stage breast carcinoma. Am J Surg Pathol 31, 129-140 (2007)

13. G. Acs, G. Paragh, S. T. Chuang, C. Laronga and P. J. Zhang: The presence of micropapillary features and retraction artifact in core needle biopsy material predicts lymph node metastasis in breast carcinoma. Am J Surg Pathol 33, 202-210 (2009)

14. T. Bujas, I. Pavic, T. Lenicek, A. Mijic, B. Kruslin and D. Tomas: Peritumoral retraction clefting correlates with advanced stage squamous cell carcinoma of the esophagus. Pathol Oncol Res 14, 443-447 (2008)

15. D. Tomas, B. Spajic, M. Milosevic, A. Demirovic, Z. Marusic and B. Kruslin: Extensive retraction artifact predicts biochemical recurrence-free survival in prostatic carcinoma. Histopathology (In press)

16. R. H. Young, J. R. Srigley, M. B. Amin, T. M. Ulbright and A. L. Cubilla, Atlas of tumor pathology. Washington, DC: Armed Forces Institute of Pathology, Washington, DC (1998)

17. B. L. Baisden, H. Kahane and J. I: Epstein. Perineural invasion, mucinous fibroplasia and glomerulations: diagnostic features on prostate needle biopsy. Am J Surg Pathol 23, 918-924 (1999) 
18. D. G. Bostwick and P. A. Dundore, Biopsy pathology of the prostate. London: Chapmann\&Hall Medical, London (1997)

19. J. I. Epstein: Diagnostic criteria of limited adenocarcinoma of the prostate on needle biopsy. Hum Pathol 26, 223-229 (1995)

20. J. R. Durham, M. W. Lee, M. B. Amin, D. S. Shulz and W. G. Stetler-Stevenson: Clefting in adenocarcinoma of the prostate gland (abstract). Am J Clin Pathol 102, 536 (1994)

21. B. Halpert and W. R. Schmalhorst: Carcinoma of the prostate in patients 70 to 79 years old. Cancer 19, 695-698 (1966)

22. B. Halpert B, E. A. Sheehan, W. R. Schmalhorst and R. J. Scott: Carcinoma of the prostate: a survey of 5000 autopsies. Cancer 16, 736-742 (1963)

23. B. Kruslin, I. Novosel, A. Reljic, H. Cupic, B. Spajic and M. Belicza: Periacinar cleft-like spaces in prostatic needle core biopsies (abstract). Acta clin Croat 41, 175 (2002)

24. H. Rogatsch, T. Mairinger, W. Horninger, A. Gschwendtner, G. Bartsch and G. Mikuz: Optimized preembedding method improves the histologic yield of prostatic core needle biopsies. Prostate 42, 124-129 (2000)

25. H. Rogatsch, P. Moser, H. Volgger, W. Horninger, G. Bartsch, G. Mikuz and T. Mairinger: Diagnostic effect of an improved preembedding method of prostate needle biopsy specimens. Hum Pathol 31, 1102-1107 (2000)

26 P. Thorson, R. T. Vollmer, C. Arcangeli, D. W. Keetch and P.A. Humphrey: Minimal carcinoma in prostate needle biopsy specimens: diagnostic features and radical prostatectomy follow-up. Mod Pathol 11, 543-551 (1998)

27. M. Herawi, A. V. Parwani, J. Irie and J. I. Epstein: Small glandular proliferations on needle biopsies. Most common benign mimickers of prostatic adenocarcinoma sent in for expert second opinion. Am J Surg Pathol 29, 874-880 (2005)

28. J. R. Srigley: Benign mimickers of prostatic adenocarcinoma. Mod Pathol 17, 328-348 (2004)

29. G. Aus, C. C. Abbou, M. Bolla, A. Heidenreich, H. P. Schmid, H. van Poppel, J. Wolff, F. Zattoni and European Association of Urology: EAU guidelines on prostate cancer. Eur Urol 48, 546-551 (2005)

30. J. C. Chewille and D. G. Bostwick: Postatrophic hyperplasis of the prostate. A histologic mimic of prostatic adenocarcinoma. Am J Surg Pathol 19: 1068-1075 (1995)

31. A. M. De Marzo, E. A. Platz, J. I. Epstein, T. Ali, A. Billis, T. Y. Chan, L. Cheng, M. Datta, L. Egevad, D. Ertoy-Baydar, X. Farre, S. W. Fine, K. A. Iczkowski, M. Ittmann, B. S. Knudsen, M. Loda, A. Lopez-Beltran, C. Magi-Galluzzi, G. Mikuz, R. Montironi, E. Pikarsky, G.
Pizov, M. A. Rubin, H. Samaratunga, T. Sebo, I. A. Sesterhenn, R. B. Shah, S. Signoretti, J. Simko, G. Thomas, P. Troncoso, T. T. Tsuzuki, G. J. van Leenders, X. J.Yang, M. Zhou, W. D. Figg, A. Hoque and M. S. Lucia: A working group classification of focal prostate atrophy lesions. Am J Surg Pathol 30, 1281-1291 (2006)

32. K. A. Iczkowski: Current prostate biopsy interpretation. Criteria for cancer, atypical small acinar proliferation, highgrade prostatic intraepithelial neoplasia, and use of immunostains. Arch Pathol Lab Med 130, 835-843 (2006)

33. M. D. Slater, C. Lauer, A. Gidley-Baird and J. A. Barden: Markers for the development of early prostate cancer. J Pathol 199, 368-377 (2003)

34. J. E. McNeal and D. G. Bostwick: Spread of adenocarcinoma within prostatic ducts and acini. Morphologic and clinical correlations. Am J Surg Pathol 20, 802-814 (1996)

35. W. A. Sakr: Prostatic intraepithelial neoplasia. Pathol International 54, S612-S615 (2004)

36. D. G. Bostwick and J. Qian: High-grade prostatic intraepithelial neoplasia. Mod Pathol 17, 360-379 (2004)

37. B. R. Oliai, H. Kahane and J. I. Epstein: Can basal cells be seen in adenocarcinoma of the prostate?: an immunohistochemical study using high molecular weight cytokeratin (clone 34 beta E12) antibody. Am J Surg Pathol 26, 1151-1160 (2002)

38. M. Zhou, R. Shah, R. Shen and M. A. Rubin: Basal cell cocktail (34betaE12 + p63) improves the detection of prostate basal cells. Am J Surg Pathol 27, 365-371 (2003)

39. R. Parsa, A. Yang, F. McKeon and H. Green: Association of $\mathrm{p} 63$ with proliferative potential in normal and neoplastic human keratinocytes. J Invest Dermatol 113, 1099-1105 (1999)

40. S. Signoretti, W. Waltregny, J. Dilks, B. Isaac, D. Lin, L. Garraway, A. Yang, R. Montironi, F. McKeon and M. Loda: p63 is a prostate basal cell marker and is required for prostate development. Am J Pathol 157, 1769-1775 (2000)

41. M. A. Weinstein, S. Signoretti and M. Loda: Diagnostic utility of immunohistochemical staining for p63, a sensitive marker of prostatic basal cells. Mod Pathol 15, 1302-1308 (2002)

42. D. R. Rowley: What might a stromal response mean to prostate cancer progression? Cancer Metastasis Rev 17, 411-419 (1998)

43. A. Noel and J. M. Foidart: The role of stroma in breast carcinoma growth in vivo. J Mammary Gland Biol Neoplasia 3, 215-225 (1998) 
44. M. Martin, P. Pujuguet and F. Martin: Role of stromal myofibroblasts infiltrating colon cancer in tumor invasion. Pathol Res Pract 192, 712-717 (1996)

45. J. A. Tuxhorn, G. E. Ayala and D. R. Rowley: Reactive stroma in prostate cancer progression. J Urol 166, 2472$2483(2001)$

46. J. A. Tuxhorn, G. E. Ayala, M. J. Smith, V. C. Smith, T. D. Dang and D. R. Rowley: Reactive stroma in human prostate cancer: induction of myofibroblast phenotype and extracellular matrix remodeling. Clin Cancer Res 8, 29122923 (2002)

47. D. Tomas and B. Kruslin: The potential value of (Myo)fibroblastic stromal reaction in the diagnosis of prostatic adenocarcinoma. Prostate 61, 324-331 (2004)

48. D. Tomas, M. Ulamec, T. Hudolin, S. Bulimbasic, M. Belicza and B. Kruslin: Myofibroblastic stromal reaction and expression of tenascin-C and laminin in prostate adenocarcinoma. Prostate Cancer Prostatic Dis 9, 414-419 (2006)

49. Y. N. Niu and S. J. Xia: Stroma-epithelium crosstalk in prostate cancer. Asian J Androl 11, 28-35 (2009)

50. M. Augsten, C. Hagglof, E. Olsson, C. Stolz, P. Tsagozis, T. Levchenko, M. J. Frederick, A. Borg, P. Micke, L. Egevad and A. Ostman: CXCL14 is an autocrine growth factor for fibroblasts and acts as a multi-modal stimulator of prostate tumor growth. Proct Natal Acad Sci USA 106, 3414-3419 (2009)

51.A. F. Olumi, G. D. Grossfeld, S. W. Hayward, P. R. Carroll, T. D. Tlsty and G. R. Cunha: Carcinomaassociated fibroblasts direct tumor progression of initiated human prostatic epithelium. Cancer Res 59, 5002-5011 (1999)

52. F. Yang, J. A. Tuxhorn, S. J. Ressler, S. J. Mcalhany, T. D. Dang and D. R. Rowley: Stromal expression of connective tissue growth factor promotes angiogenesis and prostate cancer tumorigenesis. Cancer Res 65, 8887-8895 (2005)

53. G. Ayala, J. A. Tuxhorn, T. M. Wheeler, A. Frolov, P. T. Scardino, M. Ohori, M. Wheeler, J. Spitler and D. R. Rowley: Reactive stroma as a predictor of biochemical-free recurrence in prostate cancer. Clin Cancer Res 9, 47924801 (2003)

54. D. Tomas, B. Spajic, M. Milosevic, A. Demirovic, Z. Marusic and B. Kruslin: Intensity of stromal changes predicts biochemical recurrence-free survival in prostatic carcinoma. Scand J Urol Nephrol (In press), DOI 10.3109/00365599.2010.485578.

55. N. Yanagisawa, R. Li, D. Rowley, H. Liu, D. Kadmon, B. J. Miles, T. M. Wheeler and G. E. Ayala: Stromogenic prostatic carcinoma pattern (carcinomas with reactive stromal grade 3) in needle biopsies predicts biochemical recurrence-free survival in patients after radical prostatectomy. Hum Pathol 38, 1611-1620 (2007)

56. A. Orimo, Y. Tomioka, Y. Shimizu, M. Sato, S. Oigawa, K. Kamata, Y. Nogi, S. Inoue, M. Takahashi, T. Hata and M. Muramatsu: Cancer-associated myofibroblasts possess various factors to promote endometrial tumor progression. Clin Cancer Res 7, 3097-3105 (2001)

57. M. Orlandini and S. Oliveiro: In fibroblasts Vegf-D expression is induced by cell-cell contact mediated by cadherin-11. J Biol Chem 276, 6576-6581 (2001)

58. H. P. Erickson: Tenascin-C, tenascin-R, and tenascin$\mathrm{X}$ : a family of talented proteins in search of their functions. Curr Opin Cell Biol 5, 869-876 (1993)

59. G. Orend: Potential oncogenic action of tenascin-C in tumorigenesis. Int J Biochem Cell Biol 37, 1066-1083 (2005)

60. G. Vollmer: Biologic and oncologic implications of tenascin-C/hexabrachion proteins. Crit Rev Oncol/Hematol $25,187-210$ (1997)

61. R. Kammerer, R. Ehret and S. von Kleist: Isolated extracellular matrixbased three-dimensional in vitro models to study orthotopically cancer cell infiltration and invasion. Eur J Cancer 34, 1950-1957 (1998)

62. H. J. Merker: Morphology of the basement membrane. Microsc Res Tech 28, 95-124 (1994)

63. M. Patarroyo, K. Tryggvason and I. Virtanen: Laminin isoforms in tumor invasion, angiogenesis and metastasis. Semin Cancer Bio 12, 197-207 (2002)

64. S. Kaulfuss, S. von Hardenberg, S. Schweyer, A. M. Herr, F. Laccone, S. Wolf and P. Burfeind: Leupaxin acts as a mediator in prostate carcinoma progression through deregulation of p120catenin expression. Oncogene 28, 3971-3982 (2009)

65. M. O. Ports, R. B. Nagle, G. D. Pond and A. E. Cress: Extracellular engagement of alpha6 integrin inhibited urokinase-type plasminogen activator-mediated cleavage and delayed human prostate bone metastasis. Cancer Res 69, 5007-5014 (2009)

66. J. Pontes-Junior, S. T. Reis, M. Dall'oglio, L. C. Neves de Oliveira, J. Cury, P. A. Carvalho, L. A. Ribeiro-Filho, K. R. Moreira Leite and M. Srougi: Evaluation of the expression of integrins and cell adhesion molecules through tissue microarray in lymph node metastases of prostate cancer. J Carcinog 8, 3 (2009)

67. H. R. Contreras, R. A. Ledezma, J. Vergara, F. Cifuentes, C. Barra, P. Cabello, I. Gallegos, B. Morales, C. Huidobro and E. A. Castellon: The expression of syndecan1 and -2 is associated with Gleason score and epithelialmesenchymal transition markers, E-cadherin and betacatenin, in prostate cancer. Urol Oncol (In press) 
Retraction artifact in the prostate

68. C. J. Dimitroff, M. Lechpammer, D. Long-Woodward and J. L. Kutok: Rolling of human bone-metastatic prostate tumor cells on human bone marrow endothelium under shear flow is mediated by E-selectin. Cancer Res 64, 52615269 (2004)

69. A. Popovic, A. Demirovic, B. Spajic, G. Stimac, B. Kruslin and D. Tomas: Expression and prognostic role of syndecan-2 in prostate cancer. Prostate Cancer Prostatic Dis 13, 78-82 (2010)

70. S. Mukherjee, A. M. Richardson, J. Rodriguez-Canales, K. Ylaya, H. S. Erickson, A. Player, E. S. Kawasaki, P. A. Pinto, P. L. Choyke, M. J. Merino, P. S. Albert, R. F. Chuaqui and M. R. Emmert-Buck: Identification of EpCAM as a molecular target of prostate cancer stroma. Am J Pathol 175, 2277-2287 (2009)

71. M. Ulamec, D. Tomas, H. Cupic, T. Lenicek, D. Balicevic and B. Kruslin: Periacinar retraction clefts and D2-40 expression in prostatic carcinoma. Virchow Arch Supp1, S148 (2009)

Key Words: Prostate, Adenocarcinoma, Histologic Criteria, Retraction Clefting, Needle Core Biopsy, Review

Send correspondence to: Bozo Kruslin, Ljudevit Jurak Department of Pathology, Sestre milosrdnice University Hospital Vinogradska 29, 10000 Zagreb, Croatia, Tel: 385-13787177, Fax: 385-1-3787244, E-mail: bkruslin@kbsm.hr

http://www.bioscience.org/current/volS3.htm 